\title{
X-ray Imaging Using Single Photon Processing with Semiconductor Pixel Detectors
}

\author{
B. Mikulec ${ }^{\mathrm{a}, \mathrm{b}, *}$, M. Campbell ${ }^{\mathrm{a}}$, E. Heijne $^{\mathrm{a}}$, X. Llopart $^{\mathrm{a}}$, \\ L. Tlustos ${ }^{\mathrm{a}}$ \\ ${ }^{a}$ Medipix Collaboration, CERN, 1211 Geneva 23, Switzerland \\ ${ }^{\mathrm{b}}$ now with the University of Geneva, 30 quai Ernest-Ansermet, 1211 Geneva 4, \\ Switzerland
}

\begin{abstract}
More than 10 years experience with semiconductor pixel detectors for vertex detection in high energy physics experiments together with the steady progress in CMOS technology opened the way for the development of single photon processing pixel detectors for various applications including medical X-ray imaging. The state of the art of such pixel devices consists of pixel dimensions as small as $55 \times 55$ $\mu \mathrm{m}^{2}$, electronic noise per pixel $<100 \mathrm{e}^{-} \mathrm{rms}$, signal-to-noise discrimination levels around $1000 \mathrm{e}^{-}$with a spread $<50 \mathrm{e}^{-}$and a dynamic range up to 32 bits per pixel. Moreover, the high granularity of hybrid pixel detectors makes it possible to probe inhomogeneities of the attached semiconductor sensor.
\end{abstract}

Key words: single photon detection; quantum imaging; semiconductor detectors; $\mathrm{X}$-ray imaging; Medipix

\section{Introduction}

Hybrid semiconductor detectors represent the basic tools to perform particle tracking in the vertex region in high energy physics experiments. The introduction of silicon strip detectors into this field dates back to 1980 [1], whereas silicon pixel detectors appeared at the beginning of the 90's [2]. Silicon sensors have considerably improved since then, but the continuing popularity of

Email address: bettina.mikulec@cern.ch (B. Mikulec). 
semiconductor detectors can be accredited mainly to progress in microelectronics. With reduced feature size the density of electronic components per unit area increases exponentially with time allowing increased functionality per unit area and/or reduced pixel size. With deep sub-micron technology it is currently possible to squeeze about 200000 transistors into an area of 1 $\mathrm{mm}^{2}$.

Small pixel sizes and correspondingly tiny input capacitance together with high electronics component densities, optimized transistors and chip design opened the new field of quantum processing for imaging applications [3,4]. Quantum processing implies that a particle signal can be distinguished from background noise. This noise discrimination is implemented with the help of a noise reducing pre-amplifier shaper circuit and a discriminator in each readout channel where it must be possible to set the discrimination level safely above the noise level. With steadily shrinking pixel sizes this concept gets more complicated as the signal charge from one particle might be shared between a number of pixels. As a consequence, a solution should be found to sum up the charge fractions belonging to the charge deposition of one particle and compare the summed charge to a threshold [5].

The simplest example of quantum processing is photon counting; as soon as the processed signal passes the threshold the counter value of the readout channel is incremented. However there is a great potential to be explored with more elaborated ways of processing single photon signals. Some projects start to use already two thresholds to select an energy band out of a continuous spectrum or perform subtraction images with one X-ray illumination (see references in [3]). For the future one can imagine to include a multi-bit ADC in each readout cell to provide 'colour' X-ray images $[3,6]$. Then it would also become possible to weight each of the ADC channels with its ideal energy weighting factor to increase the image contrast [7]. Noise discrimination is absolutely necessary to do low-dose or low-rate imaging, but it seems evident by now that energy information is ultimately wanted as well.

\section{Detector requirements in medical imaging applications}

In many aspects detector requirements for medical imaging applications are very similar to high energy physics applications. These include the need for high true 2-dimensional spatial resolution and high sensitivity, but medical imaging should deliver in addition the lowest possible dose to the patient.

In general detector requirements depend strongly on the imaging task. Therefore detectors (sensors and electronics) should be optimized for different imaging applications. Protein crystallography, angiography, mammography or $\gamma$ ray imaging may have differing and sometimes conflicting requirements. As mammography has some of the most stringent specifications within all medi- 
cal imaging fields it was chosen as an example to illustrate the requirements for a dedicated imaging detector:

(1) High spatial resolution: the required spatial resolution is different for analog and digital systems. Analog systems must comply with a spatial resolution of $15-20 \mathrm{lp} / \mathrm{mm}^{1}$, whereas $5-13 \mathrm{lp} / \mathrm{mm}$ for digital systems seem to be equivalent (see next point). High spatial resolution is necessary to detect tiny micro-calcifications, early indicators of breast cancer. This point is addressed in hybrid semiconductor detector development on one hand through steadily evolving miniaturization and on the other hand through direct detection of the X-rays in the semiconductor sensor without conversion layer (as used with CCD's, film and most flat panel imagers) to avoid image blurring.

(2) High contrast resolution $<3 \%$ : Contrast resolution is the greatest advantage of digital imaging systems compared to analog ones. Especially in regions of low detector illumination film is limited by the so-called fog level in the toe region of its characteristic curve. Superior contrast resolution is expected to be most beneficial for cancer detection in radiodense breasts (e.g. for younger women). Quantum processing can improve contrast resolution considerably.

(3) Uniform response: related to the previous point; for hybrid detectors this point concerns in particular the sensors (see section 4).

(4) Patient dose <3mGy: To achieve low patient dose without information loss it is necessary to have imaging systems with high $\mathrm{DQE}^{2}$ over a wide spatial frequency range. This implies high absorption and charge collection efficiency of the sensor as well as good noise performance of the readout chain. Direct detection and quantum processing are beneficial as well [7-9]. It is evident that the system has to possess as well sufficient counting rate capability.

(5) Image area $18 \times 24(24 \times 30) \mathrm{cm}^{2}$ : This is still an unsolved problem for hybrid pixel detectors. One solution adopted especially with strip detectors is a scanning slit system with a linear detector covering one dimension. Pixel chips can now be designed to be 3 -side buttable which enables to make continuous arrays of pixel detectors along 3 sides. The forth side is still needed for I/O. To solve this problem it might be possible in the future to make use of a technique increasingly popular in microelectronics industry, which is to etch holes through the readout chip to route power supply and other signal lines.

(6) Digital system

(7) Compactness, simple handling, stable operation, no cooling...

(8) Cheap

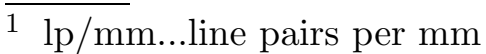

2 DQE...detective quantum efficiency 


\section{The Medipix single photon processing ASICs}

The Medipix1 chip was the first full-size photon counting ASIC and was designed with mainly mammography and dental imaging in view [10]. It emerged from experience gained with the design and the application of pixel chips for high energy physics experiments; parts of the front-end of the Medipix1 chip were even taken over from the Omega3/LHC1 pixel chip. The Medipix1 chip has been extensively studied over the years leading to the design of an improved version of the chip, Medipix2, within the framework of the Medipix2 collaboration [5,11]. Just recently, Medipix2 arrived back from the foundry as well as the first Medipix2/Si assemblies. First electrical measurement results show that the chip is working in agreement with the simulations [12]. Table 1 shows a comparison between Medipix1 and Medipix2.

\begin{tabular}{|c|c|}
\hline Medipix1 & Medipix2 \\
\hline square pixels; $170 \mu \mathrm{m}$ at the side & square pixels; $55 \mu \mathrm{m}$ at the side \\
\hline $64 \times 64$ pixels $(4096)$ & $256 \times 256$ pixels $(65536)$ \\
\hline sensitive to pos. input charge & $\begin{array}{l}\text { sensitive to pos. and neg. input charge; } \\
\text { free choice of sensor material }\end{array}$ \\
\hline $\begin{array}{c}\text { sensor leakage current compensation } \\
\text { columnwise }\end{array}$ & $\begin{array}{l}\text { pixel-by-pixel sensor leakage current } \\
\text { compensation }\end{array}$ \\
\hline one discriminator & $\begin{array}{l}\text { two discriminators (energy window) } \\
\text { designed to be linear over a wide range }\end{array}$ \\
\hline 15-bit counter per pixel & 13-bit counter per pixel \\
\hline \multirow[t]{3}{*}{$\begin{array}{c}\text { max. counting rate } \approx 1 \mathrm{MHz} / \text { pixel } \\
\left(3.5 \mathrm{GHz} / \mathrm{cm}^{2}\right)\end{array}$} & $\begin{array}{c}\text { max. counting rate } \approx 1 \mathrm{MHz} / \text { pixel } \\
\left(33 \mathrm{GHz} / \mathrm{cm}^{2}\right)\end{array}$ \\
\hline & $\begin{array}{c}3 \times 3 \text { matrix of pixels with analog } \\
\text { output to study charge sharing }\end{array}$ \\
\hline & 3-side buttable \\
\hline parallel I/O & serial or parallel I/O \\
\hline $\begin{array}{c}1 \mu \mathrm{m} \text { SACMOS technology } \\
\text { (1.6M transistors/chip) }\end{array}$ & $\begin{array}{c}0.25 \mu \mathrm{m} \text { technology } \\
\text { (33M transistors/chip) }\end{array}$ \\
\hline
\end{tabular}

Table 1

Comparison between the Medipix1 and the Medipix2 single photon processing pixel chips. 
In fig. 1 (left) an image of a $300 \mu \mathrm{m}$ thick copper mask was taken with a ${ }^{109} \mathrm{Cd}$ source and one of the first bump-bonded Medipix2/Si assemblies to give an impression of the tiny pixel size. The chip used for this image came from the first production run which showed a yield problem resulting in some dead columns. Moreover, during bump-bonding the sensor was shifted by three rows with respect to the chip. This misalignment is visible as 3 dead rows at the bottom of the image and means that the guardring of the sensor was not connected. Neglecting these problems, the resulting image is very promising.

As a comparison an image of the same object under similar measurement conditions was made with a Medipix1/Si assembly (see right fig. 1$)^{3}$. Due to the larger pixel size the number of counts/pixel is higher, but the contour of the letters is much worse.
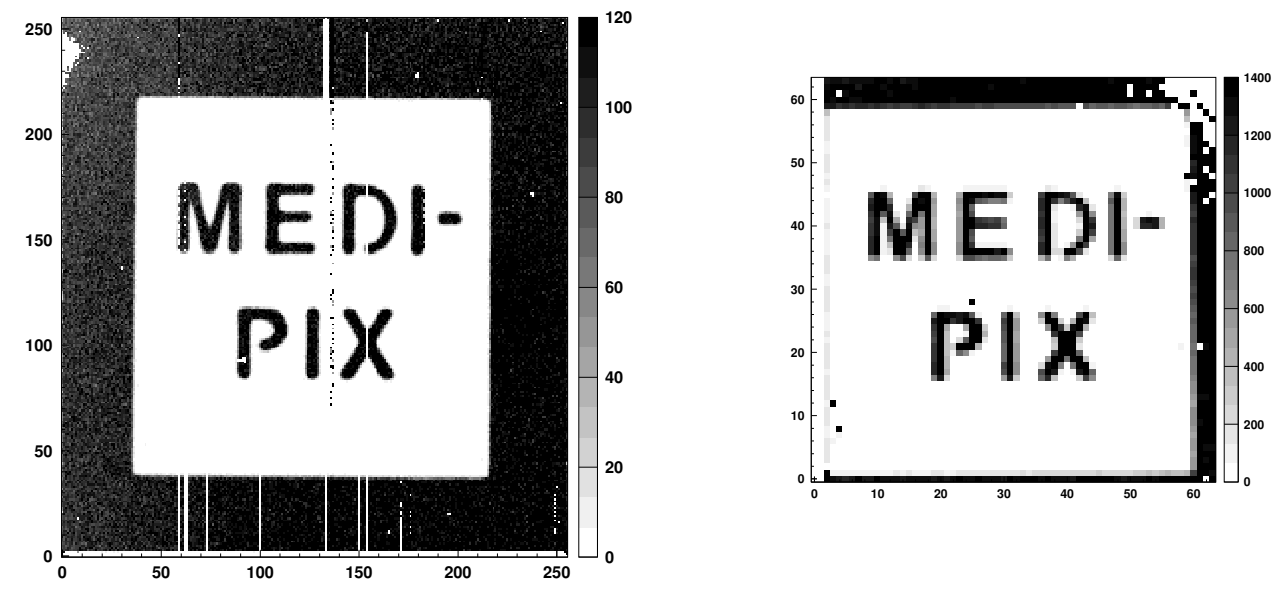

Fig. 1. Image of a copper mask taken with a ${ }^{109} \mathrm{Cd}$ source; the left image comes from a Medipix2/Si assembly, the right one from a Medipix1/Si assembly.

At present, Medipix2 is the single photon processing pixel chip with the smallest pixel size and the highest counting rate per unit area. It will offer the possibility to study optimal pixel sizes for different applications and the implication of charge sharing effects to DQE and contrast resolution.

\section{Low and high frequency noise in silicon detectors}

For medical imaging applications it is imperative to reach a high DQE as mentioned before. The DQE describes how the SNR at the input of the imaging system $\left(\mathrm{SNR}_{\text {in }}\right)$ is transferred to the output $\left(\mathrm{SNR}_{\text {out }}\right)$ and can be written as

$\overline{3}$ The top right corner of the assembly shows some bump-bonding problems. 
$\mathrm{SNR}_{\text {out }}^{2} / \mathrm{SNR}_{\text {in }}^{2}$. Taking uniform illuminations of several Medipix1/Si assemblies (flood images) it was seen that the SNR defined in this case simply by the fraction (mean number of counts/sigma of the count distribution) quickly flattens out at values around 20 instead of following a square root dependence predicted by Poisson statistics. Nevertheless, applying a flat field correction resulted in a curve representing the theoretically predicted limit over the full dynamic range of the chip and reaching values of SNR $>160$ [3]. The flat field correction is a correction method applied routinely in the imaging field. It consists of multiplying each imaging element with its calibration or 'efficiency' factor attributed beforehand with a certain number of flood fields. Investigation were therefore started to understand the reasons for the relatively large spread in counts under uniform illumination without flat field correction.

Several observations were made and are summarized below $[3,13,14]$ :

(a) Low spatial frequency patterns: The silicon detectors show a 'wave' pattern of higher and lower counting regions, in particular visible while working in under-depletion. These 'waves' of period of the order of $1 \mathrm{~mm}$ move slightly increasing the detector bias voltage from a few volts until the depletion voltage $(\approx 20 \mathrm{~V}$ in our case). Increasing the bias voltage further, the location of the waves is stable and the amplitude decreases. Nevertheless, even at voltages above $100 \mathrm{~V}$ the wave structure is still visible with count variations around $\pm 2 \%$. This low frequency noise has been ascribed to bulk inhomogeneities due to doping fluctuations during crystal growth (see [15]). As it is stable in time it can be corrected for with a flat field correction made at the corresponding detectors bias voltage.

(b) High spatial frequency pattern: Besides the macroscopic fluctuations mentioned in (a) a fixed pattern noise locally varying from pixel to pixel has been observed. This high frequency noise is independent in its location from the applied bias. A flat field map made even in under-depletion can smoothen the pixel-to-pixel variations of an image taken in overdepletion. The reason for this observed high frequency noise is still under investigation. Possible explanations might be variations in the pixel geometry and/or electric field components parallel to the pixel surface due to doping inhomogeneities, which lead to differences in charge collection, or threshold variations.

(c) Energy dependence of the flat field correction: It has been observed that the flat field correction was ineffective in the case of an image taken with a mammographic phantom (objects embedded in $4 \mathrm{~cm}$ thick PMMA) and a Mo X-ray tube (spectral X-ray source) where the flat field was calculated in the absence of any PMMA. Due to the higher absorption probability of low energy X-rays in the PMMA (or the patient) compared to X-rays from the upper end of the spectrum, the X-ray spectrum incident on the detector is hardened. This results in a different mean conversion depth of the X-rays in the sensor leading to a different count distribution on the pixel matrix. Under real medical imaging conditions this represents 
a problem as it might result in image artifacts and false diagnosis. Fortunately it was shown that an interpolated flat field map could effectively correct the data [14]. Thus, provided the thickness and density of the imaged object is known an appropriate flat field correction can be used.

To conclude, it has been shown that single photon pixel detectors with small pixel size can be used as well to probe the connected semiconductor sensor. Even silicon sensors show pronounced low and high spatial frequency patterns in the count distribution of a uniform illumination. Due to the energy dependence of some correction methods used for imaging applications it would be desirable to improve the sensor uniformity.

\section{Conclusions}

Single photon processing is becoming more and more popular for various kinds of imaging applications due to its advantages compared to charge integrating systems. It has a huge and still unexploited potential as use of the energy information may lead to 'colour' X-ray imaging.

\section{References}

[1] E.H.M. Heijne, L. Hubbeling, B.D. Hyams, P. Jarron, P. Lazeyras, F. Piuz, J.C. Vermeulen and A. Wylie, A silicon surface barrier microstrip detector designed for high energy physics, Nucl. Instr. Meth. 178 (1980) 331-343 and CERN/ECP 91-26

[2] F. Anghinolfi, P. Aspell, K. Bass, W. Beusch, L. Bosisio, C. Boutonnet, P. Burger, M. Campbell, E. Chesi, C. Claeys, J.C. Clemens, M. Cohen Solal, I. Debusschere, P. Delpierre, D. Di Bari, B. Dierickx, C.C. Enz, E. Focardi, F. Forti, Y. Gally, M. Glaser, T. Gys, M.C. Habrard, E.H.M. Heijne, L. Hermans, R. Hurst, P. Inzani, J.J. Jäger, P. Jarron, F. Krummenacher, F. Lemeilleur, V. Lenti, V. Manzari, G. Meddeler, M. Morando, A. Munns, F. Nava, F. Navach, C. Neyer, G. Ottaviani, F. Pellegrini, F. Pengg, R. Perego, M. Pindo, R. Potheau, E. Quercigh, N. Redaelli, L. Rossi, D. Sauvage, G. Segato, S. Simone, G. Stefanini, G. Tonelli, G. Vanstraelen, G. Vegni, H. Verweij, G.M. Viertel and J. Waisbard, A 1006 element hybrid silicon pixel detector with strobed binary output, IEEE Trans. Nucl. Sci. NS-39 (1992) 654-661

[3] B. Mikulec, Development of Segmented Semiconductor Arrays for Quantum Imaging, presented at the Samba2 conference, May 27-29 2002, Trieste, Italy, submitted for publication in NIM A 
[4] M. Campbell, Electronics for pixel detectors, Proc. of the 7th Workshop on Electronics for LHC Experiments, 10-14 September 2001, Stockholm, Sweden

[5] X. Llopart, M. Campbell, R. Dinapoli, D. San Segundo and E. Pernigotti, Medipix2, a 64k pixel readout chip with $55 \mu \mathrm{m}$ square elements working in single photon counting mode, IEEE Trans. Nucl. Sci., vol. 49, 2279-2283, October 2002

[6] S. Cadeddu, C. Caligiore, M. Caria, A. Lai, D. LoPresti, S. Panebianco, C. Petta, P. Porcu, N. Randazzo, S. Reito and G.V. Russo, The design of a system for coloured digital radiology with VLSI circuits and GaAs pixel detectors, NIM A 419 (1998) 270-275

[7] R.N. Cahn, B. Cederström, M. Danielsson, A. Hall, M. Lundqvist and D. Nygren, Detective quantum efficiency dependence on x-ray energy weighting in mammography, Med. Phys., 26(12), December 1999, 2680-2683

[8] J. Watt, D.W. Davidson, C. Johnston, C. Smith, L. Tlustos, B. Mikulec, K.M. Smith and M. Rahman, Dose reductions in dental X-ray imaging using Medipix, presented at the 6th International Conference on Position Sensitive Detectors, September 9-13 2002, Leicester, England, accepted for publication in NIM A

[9] D.W. Davidson, J. Watt, L. Tlustos, B. Mikulec, M. Campbell, K. Mathieson, V. O'Shea, K.M. Smith and M. Rahman, Detective Quantum Efficiency of the Medipix Pixel Detector, presented at the IEEE NSS/MIC conference, Norfolk, Virginia, November 10-16 2002, to appear in the Conference Records

[10] M. Campbell, E.H.M. Heijne, G. Meddeler, E. Pernigotti and W. Snoeys, Readout for a $64 \times 64$ pixel matrix with 15-bit single photon counting, IEEE Trans. Nucl. Sci. 45 (3) (1998) 751-753 and CERN-ECP/97-010

[11] The Medipix2 Collaboration consists of 16 European institutes; see http://medipix.web.cern.ch/MEDIPIX/

[12] X. Llopart and M. Campbell, First test measurements of a $64 \mathrm{k}$ pixel readout chip working in single photon counting mode, presented at the IWORID conference 2002, Amsterdam, The Netherlands, submitted for publication in NIM A

[13] L. Tlustos, D. Davidson, M. Campbell, E. Heijne and B. Mikulec, Fixed pattern deviations in Si pixel detectors measured using the Medipix1 readout chip, presented at the IWORID conference 2002, Amsterdam, The Netherlands, submitted for publication in NIM A

[14] D.W. Davidson, C. Frödjh, V. O'Shea, H.-E. Nilsson and M. Rahman, Limitations to Flat-Field Correction Methods when using an X-ray Spectrum, presented at the IWORID conference 2002, Amsterdam, The Netherlands, submitted for publication in NIM A

[15] W. von Ammon and H. Herzer, The Production and Availability of High Resistivity Silicon for Detector Application, Nucl. Instr. Meth. 226 (1984) 94-102 